\title{
Correction to "Optimally Joint Subcarrier Matching and Power Allocation in OFDM Multihop System"
}

\author{
Lijie Wang, Yong Xi, and Jibo Wei \\ National University of Defense Technology, Changsha 410071, China \\ Correspondence should be addressed to Lijie Wang, lilywang_gao@hotmail.com
}

Received 22 June 2010; Accepted 1 August 2010

Copyright (C) 2010 Lijie Wang et al. This is an open access article distributed under the Creative Commons Attribution License, which permits unrestricted use, distribution, and reproduction in any medium, provided the original work is properly cited.

In the above paper [1], the author gives that $\partial R_{\mathrm{tot}, k} / \partial x_{k}$ is always greater than 0 as (15) in [1]. However, $\partial R_{\text {tot }, k} / \partial x_{k}$ should be

$$
\begin{aligned}
& \frac{\partial R_{\mathrm{tot}, k}\left(P_{1^{\prime}}, P_{2^{\prime}}\right)}{\partial x_{k}} \\
& =\frac{B}{4 \operatorname{In} 2} \\
& \times \frac{2 x_{k} P_{1^{\prime}}\left(P_{\mathrm{tot}}-P_{1^{\prime}}\right)+\sigma_{2}^{2}\left[P_{\mathrm{tot}}\left(H+x_{k}\right)^{2}-2 P_{1^{\prime}}\left(H^{2}+x_{k}^{2}\right)\right]}{\left[\sigma_{2}^{2}\left(H+x_{k}\right)^{2}+P_{1^{\prime}}\left(H+x_{k}\right)\right]\left[\sigma_{2}^{2}\left(H-x_{k}\right)^{2}+\left(P_{\mathrm{tot}}-P_{1^{\prime}}\right)\left(H-x_{k}\right)\right]} \\
& =\frac{B}{4 \operatorname{In} 2} \\
& \times \frac{2 x_{k} P_{1^{\prime}}\left(P_{\mathrm{tot}}-P_{1^{\prime}}\right)+\sigma_{2}^{2}\left[P_{\mathrm{tot}}\left(H+x_{k}\right)^{2}-2 P_{1^{\prime}}\left(H^{2}+x_{k}^{2}\right)\right]}{\left(H^{2}-x_{k}^{2}\right)\left[\sigma_{2}^{2}\left(H+x_{k}\right)+P_{1^{\prime}}\right]\left[\sigma_{2}^{2}\left(H-x_{k}\right)+\left(P_{\text {tot }}-P_{1^{\prime}}\right)\right]},
\end{aligned}
$$

where $P_{\text {tot }} \geq P_{1^{\prime}} \geq 0$. It is observed that the denominators of (1) and (15) in [1] are the same and positive whereas the numerators are different. From (1), it cannot be assured that $\partial R_{\mathrm{tot}, k} / \partial x_{k}$ is always greater than 0 , for example, when $P_{1^{\prime}} \rightarrow P_{\text {tot }}, \partial R_{\text {tot }, k} / \partial x_{k}<0$. Therefore, the total channel capacity is not always monotonically increasing function of $x_{k}$ for the given power allocation, and (16) and (17) in [1] cannot be obtained. Meanwhile, the extended proposition in [1, Section 4] does not stand.

\section{References}

[1] W. Wang, S. Yan, and S. Yang, "Optimally joint subcarrier matching and power allocation in OFDM multihop system," EURASIP Journal on Advances in Signal Processing, vol. 2008, Article ID 241378, 8 pages, 2008. 